\title{
OBSERVATIONS OF ULTRACOOL WHITE DWARFS
}

\author{
B. R. OPPENHEIMER \\ Astronomy Department, University of California, Berkeley, CA 94720-3411; bro@astron.berkeley.edu \\ D. SAUMON \\ Department of Physics and Astronomy, Vanderbilt University, Nashville, TN 37235 \\ S. T. HodGKIN \\ Institute for Astronomy, Cambridge University, Madingley Road, Cambridge CB3 OHA, England, UK \\ R. F. JAMESON \\ Department of Physics and Astronomy, University of Leicester, University Road, Leicester LE1 7RH, England, UK \\ N. C. Hambly \\ Institute for Astronomy, University of Edinburgh, Blackford Hill, Edinburgh EH9 3HJ, Scotland, UK \\ G. CHABRIER \\ Ecole Normale Supérieure, CRAL (UMR 5574 CNRS), 69364 Lyon Cedex 07, France \\ A. V. Filippenko AND A. L. CoIL \\ Astronomy Department, University of California, Berkeley, CA 94720-3411 \\ AND \\ M. E. BROWN \\ Department of Geological and Planetary Sciences, California Institute of Technology, 170-25, Pasadena, CA 91125 \\ Received 2000 June 15 ; accepted 2000 November 17
}

\begin{abstract}
We present new spectroscopic and photometric measurements of the white dwarfs LHS 3250 and WD $0346+246$. Along with F351 - 50, these white dwarfs are the coolest ones known, all with effective temperatures below $4000 \mathrm{~K}$. Their membership in the Galactic halo population is discussed, and detailed comparisons of all three objects with new atmosphere models are presented. The new models consider the effects of mixed $\mathrm{H} / \mathrm{He}$ atmospheres and indicate that WD $0346+246$ and $\mathrm{F} 351-50$ have predominantly helium atmospheres with only traces of hydrogen. LHS 3250 may be a double degenerate whose average radiative temperature is between 2000 and $4000 \mathrm{~K}$, but the new models fail to explain this object.

Subject headings: stars: fundamental parameters — stars: individual (LHS 3250, WD 0346+246) white dwarfs
\end{abstract}

\section{INTRODUCTION}

The recent discovery of three white dwarfs with temperatures below $4000 \mathrm{~K}$ (Hambly, Smartt, \& Hodgkin 1997; Hambly et al. 1999; Harris et al. 1999; Hodgkin et al. 2000; Ibata et al. 2000) shows that extremely cool white dwarfs have blue colors in the infrared and may peak in flux density at wavelengths as short as $0.6 \mu \mathrm{m}$. The spectral energy distributions (SEDs) of these cool white dwarfs deviate from the blackbody spectrum by up to 4 mag in the infrared because of the presence of deep absorption due to collisionally induced dipole moments in $\mathrm{H}_{2}$ molecules (commonly referred to as $\mathrm{H}_{2}$ collision induced absorption or $\mathrm{H}_{2}$ CIA; Borysow \& Frommhold 1990). Theoretical predictions of this phenomenon by Bergeron et al. (1994), Saumon \& Jacobson (1999), and Hansen (1999) have now been unambiguously confirmed in LHS 1126 (Bergeron, Saumon, \& Wesemael 1995a), LHS 3250 (Harris et al. 1999), and WD 0346+246 (Hodgkin et al. 2000), which has been spectroscopically observed in the infrared. The SED demonstrates that the widespread assumption that optical and infrared colors monotonically redden with decreasing effective temperature is wrong.

We present here a compilation of new and previously published observations of three "ultracool" white dwarfs, WD 0346+246, LHS 3250, and F351-50. Our new observations of WD $0346+246$ and LHS 3250 include spectra and photometry from the $U$ through $K$ bands, the wave- length region in which these stars are brightest. All of these objects were discovered because of their high proper motions, and all clearly exhibit extreme flux deficits in the near-infrared. Furthermore, the measured parallaxes and proper motions enable a discussion of their membership in the Galaxy's halo population.

To complement the observational work, we present synthetic spectra of such white dwarfs. We expand the models of Saumon \& Jacobson (1999) by considering atmospheres of mixed hydrogen and helium composition. We use these new models to fit our new data along with previous parallax measurements of WD $0346+246$ (Hambly et al. 1999) and LHS 3250 (Harris et al. 1999). Such fits should ideally provide complete physical solutions for these white dwarfs. However, only the spectra of WD $0346+246$ and F351 - 50 (published in Ibata et al. 2000 without a parallax measurement) can be explained by the new models. LHS 3250 remains an enigma, but we discuss the general properties in depth. For WD $0346+246$, because the parallax is known, we derive the luminosity $(L)$, effective temperature $\left(T_{\text {eff }}\right)$, mass $(M)$, and radius $(R)$.

\section{OBSERVATIONS}

Our new observations of LHS 3250 and WD $0346+246$ spanned more than a year and involved multiple instruments and telescopes. For WD $0346+246$, we have rereduced our infrared spectrum (first presented in Hodgkin et 


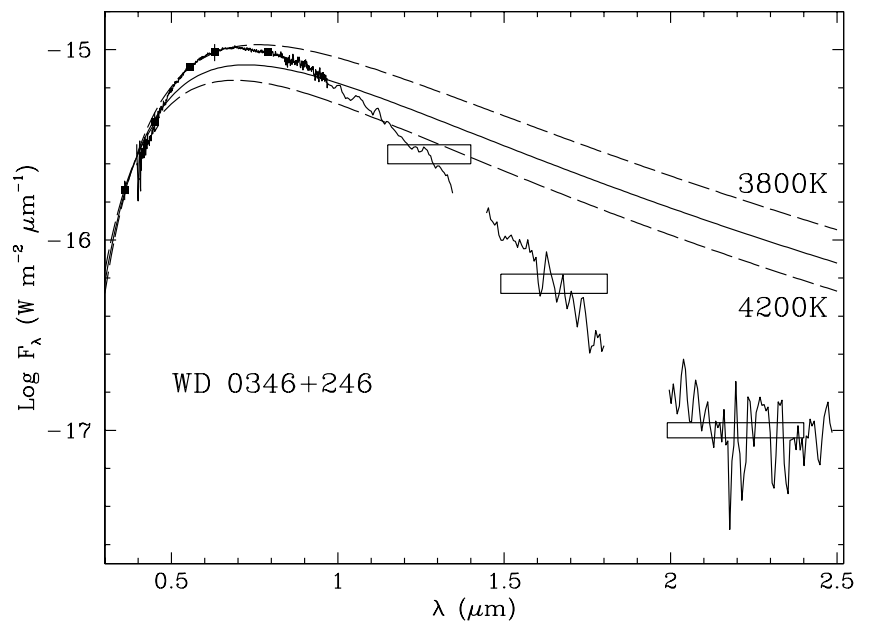

FIG. 1.-Spectra and photometry of WD $0346+246$. The open boxes indicate the infrared photometry. Their width corresponds to the wavelength range of the filter, and their height corresponds to the uncertainty in flux density. The small filled squares mark the optical photometric measurements. Those points without error bars have errors smaller than the size of the squares. The spectral data have been smoothed over 3 pixels (approximately 1 resolution element). Three blackbody spectra (at 3800 , 4000 , and $4200 \mathrm{~K}$ ) are also plotted to show the fitting used in $\S 5$ to the wavelength region below $0.5 \mu \mathrm{m}$. They also clearly illustrate the strong infrared suppression.

al. 2000), obtained a new optical spectrum, and measured $U$ - and $B$-band photometry. For LHS 3250 , we have obtained spectra spanning $0.8-2.5 \mu \mathrm{m}$. The photometric measurements are shown in Table 1. Figure 1 presents the photometry and spectroscopy of WD $0346+246$, and Figure 2 plots the new spectroscopy of LHS 3250 along with the Harris et al. (1999) spectrum and photometry. In our subsequent analysis sections, we additionally consider the spectrum of F351 - 50 from Ibata et al. (2000).

\section{1. $W D 0346+246$}

Hambly et al. (1997) presented an optical spectrum of WD $0346+246$ that fell short of reaching $1.0 \mu \mathrm{m}$. With the infrared spectrum in Hodgkin et al. (2000), a gap in the data set persisted between 0.8 and $1.0 \mu \mathrm{m}$. The Hodgkin et al. (2000) infrared spectrum seemed to indicate the presence of a sharp peak in the spectrum somewhere in this gap. The Hambly et al. (1997) spectrum was about 0.1 mag discrepant with their optical photometry as well. With this in mind, we revisited the reduction of the infrared spectrum and

TABLE 1

PHOTOMETRY OF WD $0346+246$

\begin{tabular}{lcccc}
\hline \hline Band & $\begin{array}{c}\lambda_{\text {eff }} \\
(\mu \mathrm{m})\end{array}$ & Magnitude & $\begin{array}{c}f_{\lambda} \\
\left(\mathrm{W} \mathrm{m}^{-2} \mu \mathrm{m}^{-1}\right)\end{array}$ & $\begin{array}{c}\text { Luminosity } \\
\left(L_{\odot}\right)\end{array}$ \\
\hline$U \ldots \ldots$ & 0.3686 & $20.8 \pm 0.1$ & $1.81 \times 10^{-16}$ & $5.0 \times 10^{-7}$ \\
$B \ldots \ldots$ & 0.4310 & $20.5 \pm 0.1$ & $4.14 \times 10^{-16}$ & $1.6 \times 10^{-6}$ \\
$V \ldots \ldots$. & 0.5405 & $19.06 \pm 0.01$ & $8.51 \times 10^{-16}$ & $2.5 \times 10^{-6}$ \\
$R \ldots \ldots$ & 0.6314 & $18.30 \pm 0.08$ & $1.02 \times 10^{-15}$ & $3.0 \times 10^{-6}$ \\
$I \ldots \ldots$. & 0.7927 & $17.54 \pm 0.02$ & $1.08 \times 10^{-15}$ & $4.4 \times 10^{-6}$ \\
$J \ldots \ldots$. & 1.2117 & $17.60 \pm 0.05$ & $2.79 \times 10^{-16}$ & $1.7 \times 10^{-6}$ \\
$H \ldots \ldots$ & 1.6226 & $18.2 \pm 0.1$ & $5.93 \times 10^{-17}$ & $4.5 \times 10^{-7}$ \\
$K \ldots \ldots$. & 2.1750 & $19.0 \pm 0.1$ & $9.97 \times 10^{-18}$ & $1.0 \times 10^{-7}$ \\
\hline
\end{tabular}

NoTE.-According to the synthetic spectra ( $(6)$, the total luminosity of this object is about $15 \%$ higher than the sum of the numbers in the last column.

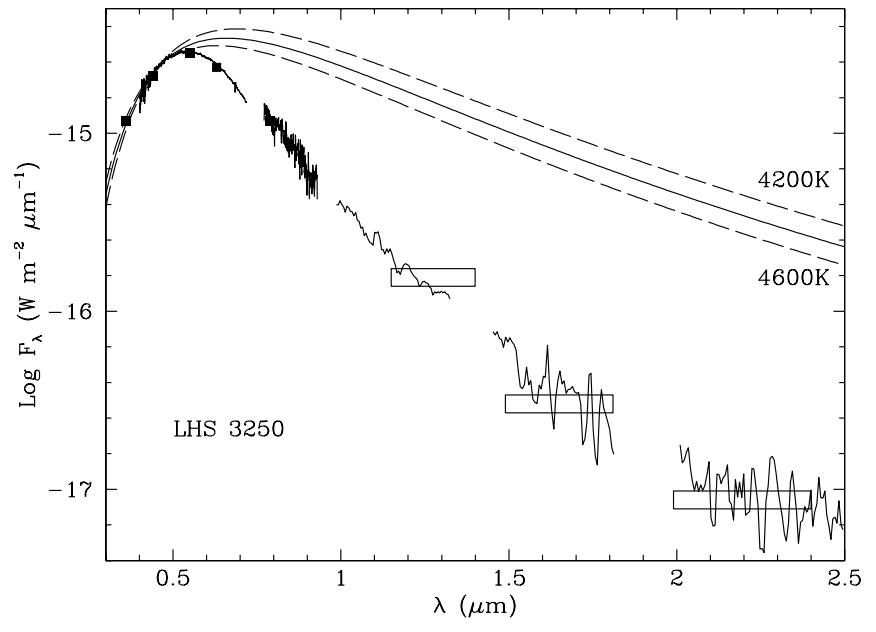

FIG. 2.- Spectra and photometry of LHS 3250. The notation and symbols are identical to those used in Fig. 1. The optical photometry and the optical spectrum come from Harris et al. (1999).

obtained a new optical spectrum with careful flux calibration independent of any imaging photometric observations.

The new reduction of the infrared spectral data previously published in Hodgkin et al. (2000; taken 1999 February at the Keck I $10 \mathrm{~m}$ telescope with the Near Infrared Camera [NIRC]; Matthews \& Soifer 1994) revealed an error in the spectrophotometric calibration that caused a peak near $1.0 \mu \mathrm{m}$. The error affected the spectrum only in the $Z_{\text {CIT }}$ band and minimally in the $J$ band. The error was due to an incomplete removal of the spectral shape of the standard star. This is a broad effect and introduced no spurious, fine features. Upon correction, the optical and nearinfrared spectra agree. Furthermore, the infrared spectrum agrees perfectly with the new, more carefully calibrated optical spectrum described below.

The new optical spectrum of WD $0346+246$ was taken with the Low-Resolution Imaging Spectrometer (LRIS; Oke et al. 1995) on the Keck II 10 m telescope on 1999 December 15 (UT) under clear conditions and 0"7 seeing. The spectrum was obtained with a low-dispersion grating and two different blocking filters to acquire the full wavelength range from 0.39 to $1.0 \mu \mathrm{m}$. The $1501 \mathrm{~mm}^{-1}$ grating blazed at $0.75 \mu \mathrm{m}$ in conjunction with the 1.0 slit provided an average dispersion of $\sim 4.8 \AA$ pixel $^{-1}$. LRIS has a single Tektronix $2048 \times 2048$ pixel CCD, which was binned in the spatial direction by a factor of 2, yielding $0.43 \mathrm{pixel}^{-1}$.

A first set of exposures was obtained with the order blocking filter GG495, which is transmissive only at wavelengths greater than $\sim 0.48 \mu \mathrm{m}$. This order blocking filter is necessary to prevent the second-order blue part of the spectrum from overlapping the first-order red spectrum. Without the blocking filter, only wavelengths shorter than about $0.74 \mu \mathrm{m}$ are uncontaminated. With the blocking filter, wavelengths from 0.48 to $0.96 \mu \mathrm{m}$ can be observed without appreciable contamination. Two exposures of $295 \mathrm{~s}$ each were taken. Immediately afterward, without repositioning the telescope, we took three internal (quartz lamp) flat-field exposures and one exposure with the Xe-Ar lamps to provide the wavelength calibration. The standard spectrophotometric calibrator HD 84937 ( $V=8.3 \mathrm{mag}, \mathrm{F} 5$ V; Oke \& Gunn 1983) was observed with the same settings to provide the spectral response of the spectrograph at an 
air mass of 1.12 , within $10 \%$ of the air mass for WD $0346+246$. The position angle for the slit was chosen to be the parallactic angle to minimize the effects of atmospheric dispersion (Filippenko 1982).

The second set of data was taken with the same grating and slit settings but without the blocking filter. Again, two $295 \mathrm{~s}$ exposures were obtained along with corresponding internal flat fields and arc lamp exposures. The same standard star was also observed in this manner.

In order to be certain that the spectrum near $1.0 \mu \mathrm{m}$ was correct and uncontaminated, we obtained service observations with the same instrument settings on 1999 December 24 (UT) but with the RG850 blocking filter installed. This transmits wavelengths redward of $\sim 0.83 \mu \mathrm{m}$, with the second-order contamination setting in at $2 \times 0.83=1.66$ $\mu \mathrm{m}$. The weather was clear, and the seeing was again $\sim 0$ ".7. One $600 \mathrm{~s}$ exposure was taken, along with internal flat fields and arc lamp exposures. Because of the low sensitivity of the $\mathrm{CCD}$ in the near-infrared, these data are very noisy.

Using IRAF, we conducted standard CCD processing and optimal spectral extraction, while flux calibration and telluric absorption band removal were done with our own routines in IDL. Final adjustments to the wavelength scale were obtained using the background sky regions.

The adopted reduction technique produces a spectrum that is flux calibrated independent of the photometric measurements. In this sense the spectrum differs from the infrared spectrum described above. The infrared spectrum is flux calibrated using the infrared photometry and the shape of a calibrator $G$ star, which is not a spectrophotometric calibrator. In that case the G star is used only to provide the correct spectral shape, while the absolute calibration is provided by the infrared photometry. The spectrum presented in Figure 1 has a signal-to-noise ratio $(\mathrm{S} / \mathrm{N})$ over several hundred through the middle of the optical region. The infrared $\mathrm{S} / \mathrm{N}$ falls from 2000 near $1 \mu \mathrm{m}$ to approximately 6 in the $K$ band.

The data taken on December 15 exhibit second-order contamination beyond $0.98 \mu \mathrm{m}$, as shown by the uncontaminated data taken on December 24. However, the infrared spectrum from NIRC has a much higher $\mathrm{S} / \mathrm{N}$, and the December 24 LRIS spectrum agrees perfectly with it, so we simply cut the optical spectrum at $0.97 \mu \mathrm{m}$. Thus, in Figure 1 , the data redward of $0.97 \mu \mathrm{m}$ are from NIRC.

The optical photometry of WD $0346+246$ presented in Hodgkin et al. (2000) and Hambly et al. (1997) was derived from CCD images made at the Jacobus Kapteyn Telescope. The $B$-band measurement provided only an upper limit, and there was no $U$-band measurement. We have subsequently obtained $U$ - and $B$-band CCD images of WD $0346+246$ with the Palomar $1.5 \mathrm{~m}$ Oscar Meyer telescope and a Tektronix $2048 \times 2048$ pixel, thinned, AR-coated CCD along with Johnson $U$ and $B$ filters. The pixel scale was 0.37 $\mathrm{pixel}^{-1}$, and the seeing on 1999 August 10 and 11 (UT) was 1".0 under photometric conditions.

On August 10 we obtained five $600 \mathrm{~s}$ exposures of the WD $0346+246$ field. Each exposure was shifted by $10^{\prime \prime}$ in one of the cardinal directions from the previous exposure to minimize the effects of bad pixels. The Landolt (1992) standard field SA 112 was also observed with two 7 s exposures such that stars 250,223 , and 275 were in the field of view.

On 1999 August 11, we observed WD 0346+246 again but with the $U$ filter. Four 1200 s exposures were made with dithering procedures similar to those described above. For
$U$-band photometric standards, we observed the Landolt (1992) field SA 110 and obtained images of stars 496, 497, $499,502,503,504,506$, and 507 in two 60 s exposures.

Data were reduced by subtracting a median of five dark frames obtained on each night and dividing by flat-field exposures made by exposing the CCD to the illuminated dome. To obtain accurate photometric measurements of the white dwarf, we averaged the measurements of all the standard stars visible in each of the two calibration fields separately.

\subsection{LHS 3250}

Harris et al. (1999) presented the optical spectrum of LHS 3250 in the range $0.4-0.7 \mu \mathrm{m}$, as well as optical and infrared photometry (reproduced in Figs. 2 and 3). To explore the SED in more detail beyond $0.7 \mu \mathrm{m}$, we obtained optical and infrared spectroscopy.

We measured the $0.75-0.98 \mu \mathrm{m}$ spectrum of LHS 3250 through service observations at the William Herschel Telescope in the Canary Islands. The low-resolution spectrum was obtained using the ISIS spectrograph with the R158R grating, giving a wavelength scale of $2.9 \AA \mathrm{pixel}^{-1}$ over 2970 $A$ on the TEK $21024 \times 1024$ pixel detector. The weather was good during the observations, and the seeing was measured to be $1^{\prime \prime}$. A $1^{\prime \prime}$ slit was employed. Two exposures, one of $1800 \mathrm{~s}$ and one of $1500 \mathrm{~s}$, were taken on the target. These were bracketed by exposures of the spectrophotometric standard Ross 640. Calibration exposures of a tungsten flat (for flat-fielding) and $\mathrm{Cu}-\mathrm{Ar}$ and $\mathrm{Cu}-\mathrm{Ne}$ arc lamps (for wavelength calibration) were also made. CCD data reduction, optimal spectral extraction, and flux calibration were performed using IRAF tasks.

We also observed LHS 3250 on 1999 July 21 (UT) using the Keck I $10 \mathrm{~m}$ telescope and NIRC (Matthews \& Soifer 1994). We obtained $Z_{\mathrm{CIT}}, J-, H$-, and $K$-band spectra spanning $1.0-2.5 \mu \mathrm{m}$.

The spectra were obtained at air mass $\sim 1.4$ during a photometric night with 0.3 seeing in $K$ band. We used two

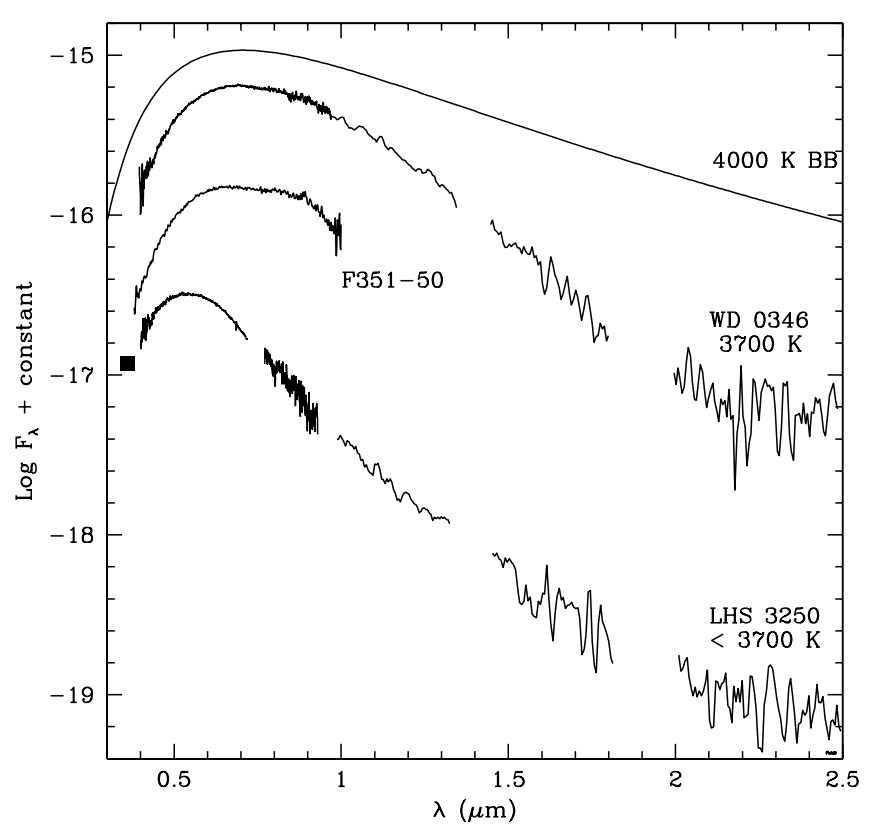

FIG. 3.-Comparison of spectra of cool halo white dwarfs; see $\S 3$ for a discussion. The optical spectrum of LHS 3250 is from Harris et al. (1999), and the F351 -50 spectrum is from Ibata et al. (2000). 
different settings. The first measured the $Z_{\mathrm{CIT}}, J$, and $H$ bands simultaneously using the $1501 \mathrm{~mm}^{-1}$ grism and the $J H$ blocking filter. The slit was 0.3 wide. We obtained four exposures of $250 \mathrm{~s}$ with the white dwarf placed at two different positions in the slit.

The $H$ - and $K$-band spectra were obtained simultaneously with the second setting. We used the same slit, the $H K$ blocking filter, and the $1201 \mathrm{~mm}^{-1}$ grism. We obtained five exposures of $200 \mathrm{~s}$ each, again placing the white dwarf in one of two slit positions for each exposure.

The F8 V star SAO $17455(V=9.58 \mathrm{mag})$ was observed with the same settings immediately after LHS 3250 to serve as a standard at an air mass of 1.4, matched to the LHS 3250 observations within $1 \%$. For the first setting we obtained two $4.1 \mathrm{~s}$ exposures and for the second setting three $4.1 \mathrm{~s}$ exposures.

Data reduction followed our standard procedure. We first subtracted pairs of images from each other to remove the sky background. Using the optimal extraction technique, we then reduced the spectra to one dimension. These steps were carried out on both the object and standard star spectra. After calibrating the wavelength scale, using the well-defined edges of the filter transmission curves (a technique good to an accuracy of $0.006 \mu \mathrm{m}$ over the band), we summed the LHS 3250 spectra and divided by the standard star spectra. This removes the pixel-to-pixel variations and accounts for the instrumental sensitivity as a function of wavelength. A model F star spectrum was then divided into the resultant spectra.

\section{SUMMARY OF OBSERVATIONS OF ULTRACOOL WHITE DWARFS}

Figures 1 and 2 and Table 1 summarize the observations from $\S 2$. To provide a means of comparison, Figure 3 shows the spectra of LHS 3250, WD 0346+246, and F351-50 (from Ibata et al. 2000). All of the white dwarf spectra are smooth and exhibit no fine features. The most important aspect of this figure is the fact that the three white dwarf spectra deviate from the blackbody spectrum redward of $0.6 \mu \mathrm{m}$. For reference, a $4000 \mathrm{~K}$ blackbody spectrum is plotted at the top of the figure. The spectra are arranged in order of what we believe to be decreasing effective temperature, from top to bottom. (See $\S 5$ for a complete discussion of this.) Figure 3 also shows that the peak wavelength appears to move toward redder wavelengths, as the temperature drops, until WD $0346+246$. Proceeding to cooler temperatures then moves the peak to bluer wavelengths. F351-50 appears on this plot because it is intrinsically faint (Ibata et al. 2000) and it exhibits a sudden change in spectral slope at $0.8 \mu \mathrm{m}$. According to models (Saumon \& Jacobson 1999; see § 6), this may be the blue edge of the second vibrational band of the $\mathrm{H}_{2}$ CIA. Other than this feature, the spectrum of F351-50 is almost identical to that of WD0346+246. The BVR colors are the same for these two objects. Without infrared photometry or a parallax it is difficult to make more concrete statements.

\section{ARE THESE WHITE DWARFS MEMBERS OF THE HALO?}

The tangential space motion of WD $0346+246$ is extremely high at $170 \mathrm{~km} \mathrm{~s}^{-1}$ and in a direction such that its Galactic orbit cannot be circular and must be strongly inclined to the Galactic disk. The conclusion in Hodgkin et al. (2000) was that this object is clearly a member of the
Galaxy's halo, regardless of what its unmeasureable radial velocity is.

Ibata et al. (2000) established that F351-50 must have a space velocity greater than $170 \mathrm{~km} \mathrm{~s}^{-1}$ and is therefore a member of the Galactic halo.

For LHS 3250, the case is not nearly as clear. Harris et al. (1999) demonstrated that the tangential velocity of LHS 3250 is $81.2 \pm 1.2 \mathrm{~km} \mathrm{~s}^{-1}$. Unfortunately, LHS 3250 is essentially at $90^{\circ}$ Galactic longitude and near the plane of the Galaxy. This, combined with the lack of any features in the spectrum that would permit measurement of the radial velocity, makes it impossible to determine whether it has a disk or halo orbit. However, there are several important features to note about the motion of LHS 3250 through the Galaxy. By subtracting the heliocentric motion from the velocities, it appears that LHS 3250 is moving toward the south Galactic pole at $73 \mathrm{~km} \mathrm{~s}^{-1}$ and toward the Galactic anticenter at $52 \mathrm{~km} \mathrm{~s}^{-1}$. Thus, the only definitive statement is that its orbit about the Galactic center cannot be circular. This makes LHS 3250 a probable member of the halo.

\section{PHYSICAL CHARACTERISTICS}

In Hodgkin et al. (2000), we derived the radius, mass, luminosity, and effective temperature of WD $0346+246$. This derivation used a technique that exploits the brightness temperature of these objects as a function of wavelength. We have conducted an extensive series of tests of the technique and have found that it is not reliable. Here we describe the technique more explicitly than before, and we also explain why it is not viable.

In Hodgkin et al. (2000), the derivation of radius, mass, and effective temperature relied upon the distance, $d$, and the assumption that the bluest part of the optical spectrum behaves as a blackbody. By fitting this region with a known blackbody spectrum, we believed that we could obtain the physical parameters. For pure $\mathrm{H}$ white dwarfs, the only known opacity in this wavelength region is due to $\mathrm{H}^{-}$, which has a weak wavelength dependence in the optical regime. In pure $\mathrm{He}$ white dwarfs, the dominant opacity source in the optical is $\mathrm{He}^{-}$, which is also very flat. The flatness of the opacity seemed to validate the technique. To support this argument, we examined plots of the brightness temperature versus $\lambda$ of synthetic spectra for pure hydrogen white dwarfs (Saumon \& Jacobson 1999). Shown in Figure 4 , these plots have nearly constant brightness temperature in the visible part of the spectrum. For example, the brightness temperature of a $T_{\text {eff }}=3500 \mathrm{~K}, \log g=8$ model, where $g$ is the surface gravity in cgs units, varies by less than $200 \mathrm{~K}$ from 0.4 to $0.9 \mu \mathrm{m}$. For white dwarfs with $T_{\text {eff }} \gtrsim 4500$ $\mathrm{K}$, the SED is quite close to that of a blackbody.

To extract the radius of the star, then, involves fitting a blackbody curve, $B(T)$, to the optical region where the brightness temperature is nearly constant (below $\lambda=0.5$ $\mu \mathrm{m})$. The fit involves two parameters, the temperature of the blackbody fit and the ratio $(R / d)^{2}$. The temperature obtained from the fit is the brightness temperature in this region of the spectrum, not $T_{\text {eff }}$. Furthermore, the brightness temperature determines the shape of the blackbody curve, while the actual flux level is determined by $(R / d)^{2}$. Figures 1 and 2 show attempts to do this fitting on WD $0346+246$ and LHS 3250 .

Using this fitted temperature, $T_{\mathrm{fit}}$, in each case, we derive the ratio $R / d$ from the following equation: $(R / d)^{2} \pi B_{U, B}\left(T_{\mathrm{fit}}\right)=F_{U, B}$, where $F_{U, B}$ is the flux in the fitted 


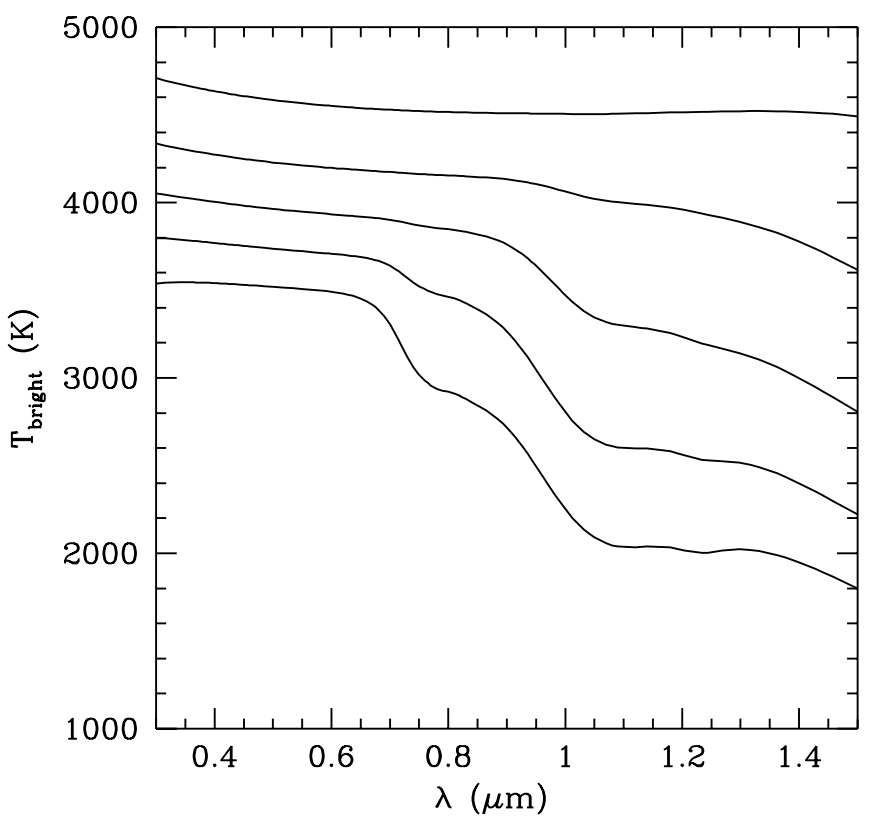

Fig. 4.-Brightness temperature as a function of wavelength from pure hydrogen synthetic spectra (Saumon \& Jacobson 1999). All models have $\log g=8$, and the effective temperature ranges from 4500 to $2500 \mathrm{~K}$ in steps of $500 \mathrm{~K}$, from top to bottom. In the cooler models, the strong drop of $T_{\text {bright }}$ in the infrared is due to $\mathrm{H}_{2}$ CIA absorption. Correspondingly, $T_{\text {bright }}$ is significantly larger than $T_{\text {eff }}$ at shorter wavelengths in these models. Note the virtual constancy of the brightness temperature at blue wavelengths.

region from $U$ to $0.5 \mu \mathrm{m}$, and $B_{U, B}\left(T_{\mathrm{fit}}\right)$ is the blackbody flux in the same wavelength region. For LHS 3250, this yields the result $R=0.014 \pm 0.002 R_{\odot}$, using the Harris et al. (1999) value of $d=30.3 \pm 0.5 \mathrm{pc}$. With the mass-radius relation from Chabrier et al. (2000), we find the mass to be 0.54 $M_{\odot}$. For WD $0346+246$, we derive $R=0.013 \pm 0.002 R_{\odot}$ and $M=0.57 M_{\odot}$.

We can now determine the effective temperature from the relation $L=4 \pi R^{2} \sigma T_{\text {eff }}^{4}$. By integrating all the flux measured from the $U$ through $K$ bands, we find for LHS 3250 $L=3.26 \times 10^{-5} L_{\odot}$ and $T_{\text {eff }}=3650 \mathrm{~K}$. Our value for $L$ is somewhat higher than that derived by Harris et al. (1999), but it includes the important region of flux at $1 \mu \mathrm{m}$, for which they had no data. For WD $0346+246$, we calculate $L=1.83 \times 10^{-5} L_{\odot}$ and $T_{\text {eff }}=3750 \mathrm{~K}$.

Criticism about the validity of this method prompted us to test it thoroughly. The white dwarf LHS 542 is an excellent test case since it has been analyzed by Leggett, Ruiz, \& Bergeron (1998) by model fitting of the SED, an optical spectrum is available (Ibata et al. 2000), and the parallax is known. Our surface brightness method predicts a radius about 50\% larger than the Leggett et al. (1998) solution. We have also performed a variety of tests using our new models (§ 6) to generate artificial, noiseless spectra (assuming $R$ and d) and comparing the results of the surface brightness method with the input parameters of the synthetic data. We find that the method works very well when the brightness temperature of the star remains fairly constant over the wavelength interval of the fit. When the (monotonic) variation of the brightness temperature exceeds $100 \mathrm{~K}$, however, the method becomes unreliable even though satisfactory fits of the spectrum can be obtained (Figs. 1 and 2). The reason is that the derived value of $T_{\text {fit }}$ over the interval is not an "average" or intermediate value in the range of brightness temperatures represented in the fitting interval. If $T_{\text {fit }}$ were an intermediate value or average of the brightness temperature, the technique would be viable. The fact is that $T_{\text {fit }}$ is systematically shifted outside of the actual range of brightness temperatures. This is due to the temperature dependence of the blackbody flux just blueward of the peak. The method thus converges to anomalous solutions for $T_{\text {fit }}$ and $R$, solutions that deviate increasingly from the actual values as the variation in brightness temperature increases. Since the degree of variation of the brightness temperature in an observed spectrum is a priori unknown, it is not possible to apply the method with confidence.

We conclude that the physical parameters of cool white dwarfs can only be obtained from fitting synthetic spectra to the observed SED.

\section{ANALYSIS OF THE PHOTOMETRY}

We can learn more about these stars by considering their locations in the Hertzsprung-Russell (H-R) diagram (Fig. 5) and in color-color diagrams (Figs. 6 and 7). Synthetic

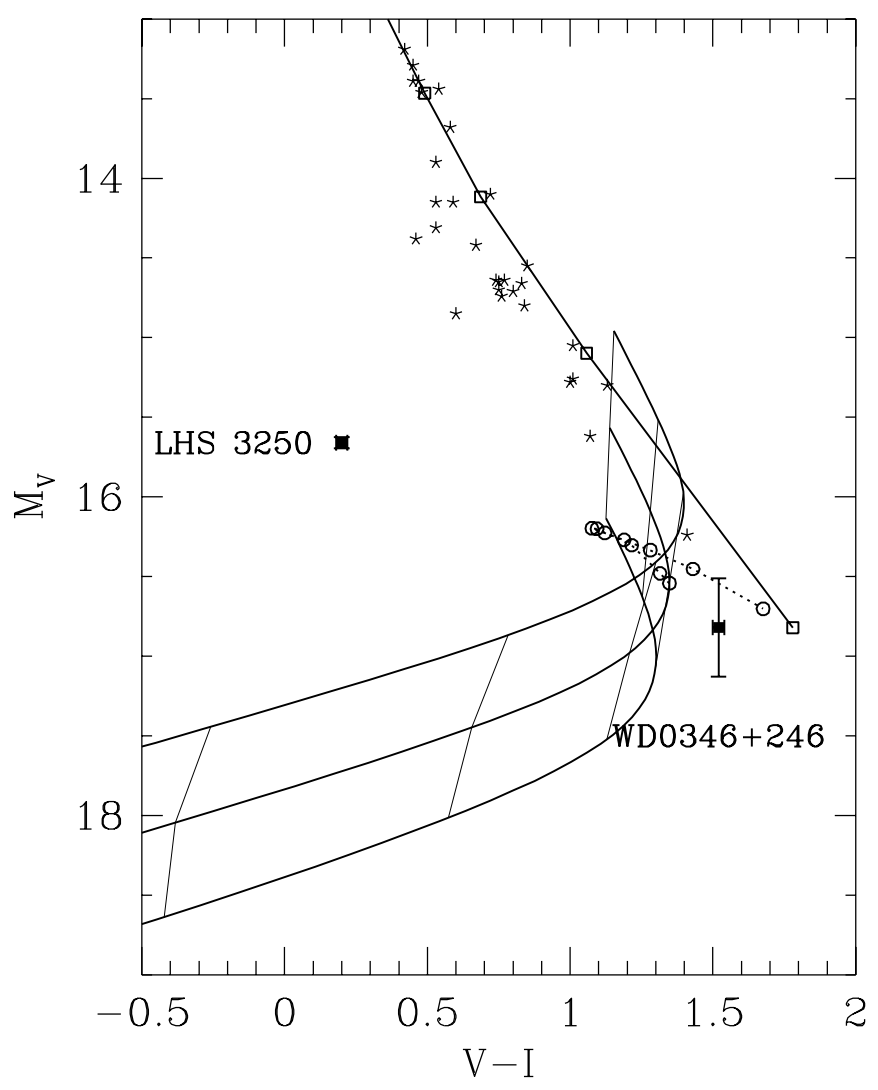

FIG. 5.-Hertzsprung-Russell diagram for cool white dwarfs. Solid lines connect the synthetic colors of pure hydrogen white dwarf atmospheres of a fixed gravity, with $\log g=7.5,8$, and 8.5 from top to bottom. Thin lines connect those models that have the same $T_{\text {eff }}$, starting with $T_{\text {eff }}=4500 \mathrm{~K}$ right of center and decreasing in steps of $500 \mathrm{~K}$. The open circles connected by a dotted line show a sequence of atmospheres with mixed hydrogen and helium composition for $T_{\text {eff }}=3500 \mathrm{~K}$ and $\log g=8$. Starting from the pure $\mathrm{H}$ model, the helium-to-hydrogen fraction $\log y=\log (N(\mathrm{He}) / N(\mathrm{H}))$ increases from -1 to 7 in steps of 1 . The colors of pure $\mathrm{He}$ atmospheres with $\log g=8$ are shown by open squares connected with a solid line. The value of $T_{\text {eff }}$ increases from 4000 to $7000 \mathrm{~K}$ in steps of $1000 \mathrm{~K}$ from bottom to top along this sequence. Star symbols represent cool, single white dwarfs with hydrogen-rich atmospheres as determined by Bergeron et al. (1997). The error bars on the $M_{V}$ values include the uncertainty in the parallaxes of the stars. 


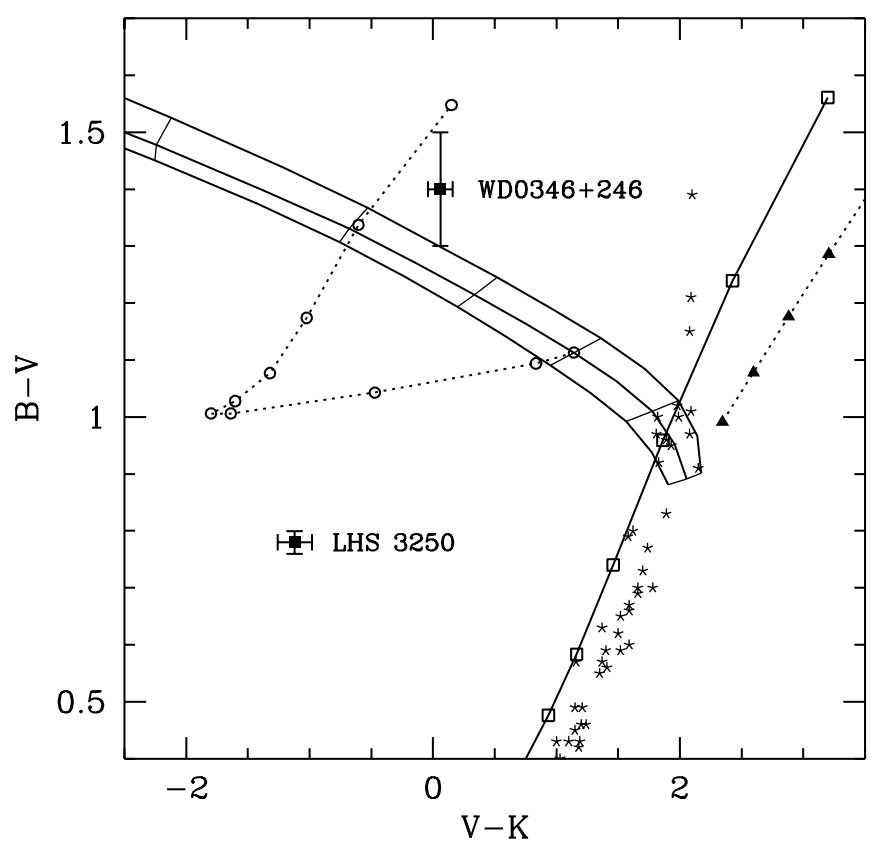

Fig. 6.-Color-color diagram for cool white dwarfs. See Fig. 5 for an explanation of the symbols. The effective temperature along the hydrogen model grid decreases from $4500 \mathrm{~K}$ right of center to $2000 \mathrm{~K}$ toward the left in steps of $500 \mathrm{~K}$. The colors of pure He atmospheres with $\log g=8$ are shown by open squares. $T_{\text {eff }}$ increases in steps of $500 \mathrm{~K}$ starting from 4000 $\mathrm{K}$ at the top. Triangles connected by a dotted line show the colors of blackbodies starting with $T=4500 \mathrm{~K}$ at the bottom and decreasing in steps of $250 \mathrm{~K}$. Compare with Fig. 15 of Bergeron, Wesemael, \& Beauchamp (1995b) or Fig. 9 of Bergeron et al. (1997).

spectra for white dwarf atmospheres of pure hydrogen and pure helium composition provide the means to interpret these diagrams. In Figures 5-7, we show a grid of pure hydrogen atmospheres (solid lines) with $\log g=7.5,8$, and

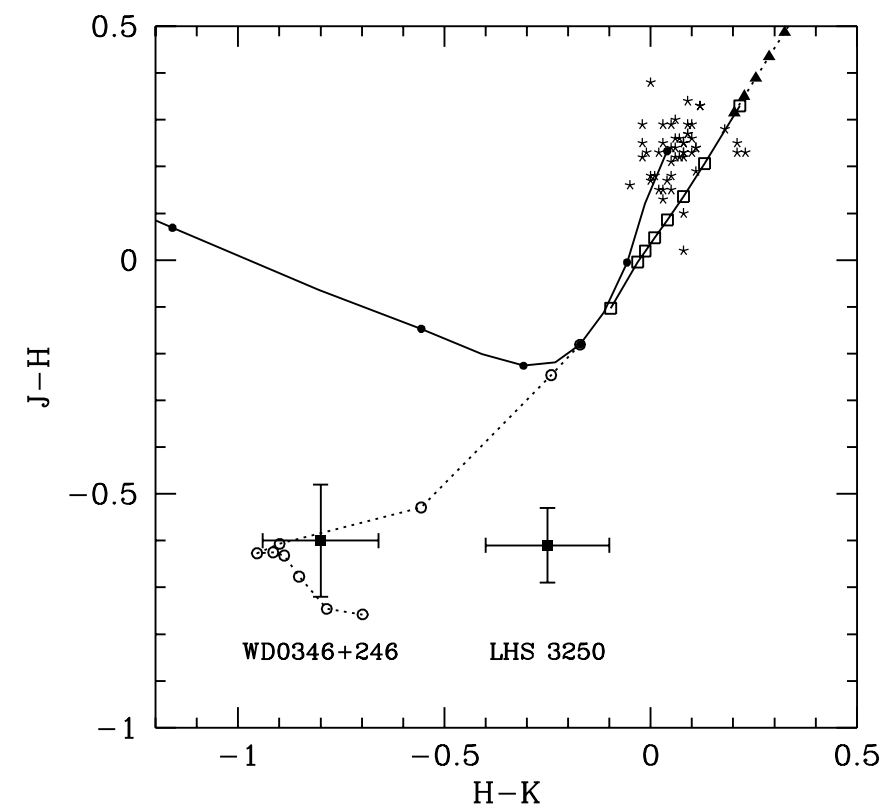

FIG. 7.-Color-color diagram for cool white dwarfs. Notation is the same as in Fig. 5. Pure H models of different gravities very nearly overlap in this diagram, so we show only the $\log g=8$ sequence for clarity. Filled circles indicate models with $T_{\text {eff }}=4000 \mathrm{~K}$ (upper right) and decreasing in steps of $500 \mathrm{~K}$. Starting from the upper right, the open squares show the pure He sequence for $T_{\text {eff }}=4000-10,000 \mathrm{~K}$ in steps of $1000 \mathrm{~K}$ and $T_{\text {eff }}=$ $20,000 \mathrm{~K}$ at the top right.
8.5 (Saumon \& Jacobson 1999), a sequence of pure helium atmospheres with $\log g=8$ (Bergeron et al. 1995a; open squares connected by a solid line), and stars from the cool white dwarf sample of Bergeron, Ruiz, \& Leggett (1997), which they identified as having a hydrogen-rich composition and which are not known as or suspected of being binary (star symbols). Since very cool white dwarfs cool at constant radius, the sequences of constant gravity models shown in Figures 5-7 also represent cooling tracks. Along these pure $\mathrm{H}$ tracks, we can associate ages using new cooling models (Chabrier et al. 2000). Table 2 gives the ages for the intersection points on the solid line grid in Figure 5.

Because neither WD $0346+246$ nor LHS 3250 are explained by the pure $\mathrm{H}$ models, we found it necessary to consider models with mixed $\mathrm{H} / \mathrm{He}$ atmospheres (Figs. 5-7, open circles and dotted lines). These preliminary models have fixed values of $T_{\text {eff }}$ and $g$ and variable values of the relative abundance of $\mathrm{He}(y=N(\mathrm{He}) / N(\mathrm{H}))$. These models allow the exploration of trends resulting from the general effects of mixed composition.

The qualitative behavior of the mixed $\mathrm{H} / \mathrm{He}$ atmospheres can be deduced from Figure 15 of Bergeron et al. (1995a), which shows $B-V$ versus $V-K$ for sequences of pure $\mathrm{H}$, pure $\mathrm{He}$, and mixed $\mathrm{H}$ and $\mathrm{He}$ composition with $y \leq 10$. As $\mathrm{He}$ is added to a cool $\mathrm{H}$ atmosphere of a given $T_{\text {eff }}$ and surface gravity, the fractional abundance of $\mathrm{H}^{-}$decreases, the relative importance of $\mathrm{He}-\mathrm{H}_{2}$ CIA opacity increases, and $V-K$ becomes bluer. This trend must reverse itself when $y$ becomes very large, however, since both $V-K$ and $B-V$ of pure He models are very red (Fig. 6). A sequence of models of fixed $T_{\text {eff }}$ and $g$ with composition varying from pure $\mathrm{H}$ to pure $\mathrm{He}$ is expected to become first very blue in $V-K$ at roughly constant $B-V$ and then turn over to redder $B-V$ and $V-K$ until it reaches the colors of a pure He atmosphere.

Our preliminary sequence of mixed composition atmospheres varies the value of $y$ over $0.1 \leq y \leq 10^{7}$ for a model with fixed $T_{\text {eff }}=3500 \mathrm{~K}$ and $\log g=8$. This sequence is indicated by the open circles connected by dotted lines in Figures 5-7. These models are rather crude in their treatment of the chemical equilibrium, and they ignore the nonideal contributions to the equation of state that are significant for pure He atmospheres (Bergeron et al. 1995a). Nevertheless, they illustrate the qualitative behavior of mixed atmospheres and are adequate for the present purpose. It is readily apparent that the $\mathrm{H}_{2}$ CIA reaches

TABLE 2

Ages of Pure H, Ultracool White Dwarfs

\begin{tabular}{cccc}
\hline \hline $\begin{array}{c}T_{\text {eff }} \\
(\mathrm{K})\end{array}$ & $\log g=8.5$ & $\log g=8.0$ & $\log g=7.8$ \\
\hline $4500 \ldots \ldots$ & 11.7 & 8.6 & 6.0 \\
$4000 \ldots \ldots$ & 12.2 & 10.0 & 7.7 \\
$3500 \ldots \ldots$ & 12.6 & 11.1 & 9.2 \\
$3000 \ldots \ldots$ & 13.0 & 12.5 & 10.7 \\
$2500 \ldots \ldots$ & 13.3 & 13.6 & 12.1 \\
$2000 \ldots \ldots$ & 13.5 & 14.8 & 13.7 \\
\hline
\end{tabular}

Notes.-Ages are given in Gyr and correspond to the intersection points in the grid of solid lines in Fig. 5. The last column corresponds to $\log g=7.8$, while the top line in Fig. 5 is for $\log g=7.5$. All of these ages are the result of new calculations (Chabrier et al. 2000) and are only pertinent to pure $\mathrm{H}$ atmospheres. 
maximum strength for $\log y \approx 2-3$. However, the colors of models of very large $\mathrm{He}$ abundance still differ significantly from those of pure $\mathrm{He}$ models because of a residual $\mathrm{H}_{2}-\mathrm{He}$ CIA opacity and $\mathrm{He}^{-}$free-free opacity enhanced by the ionization of $\mathrm{H}$. We find that in the $B V K$ color-color diagram (Fig. 6), very cool white dwarfs with mixed $\mathrm{H} / \mathrm{He}$ atmospheres occupy a much wider region than, and overlap with, the pure $\mathrm{H}$ atmospheres. Also, very cool white dwarfs with $J-H \lesssim-0.25$ have atmospheres of mixed $\mathrm{H} / \mathrm{He}$ composition (Fig. 7) and do not fall between the pure $\mathrm{H}$ and pure He sequences.

Figures 5 and 6 show that white dwarfs with atmospheres of pure $\mathrm{He}$ and pure $\mathrm{H}$ nearly overlap down to $T_{\text {eff }} \approx$ $3500-4000 \mathrm{~K}$. At these low values of $T_{\text {eff }}$, the CIA of $\mathrm{H}_{2}$ becomes very strong and the hydrogen sequence turns over (Hansen 1999; Saumon \& Jacobson 1999). The H-rich stars of Bergeron et al. (1997) reach down to the split between the two sequences. In the infrared (Fig. 7), however, the two sequences overlap only for very different ranges of $T_{\text {eff }}$ and are therefore easily distinguished. The H-R diagram (Fig. 5) is useful in assessing the evolutionary state of our objects, while the $B V K$ diagram (Fig. 6) provides a measure of the overall shape of the SED. Because CIA by $\mathrm{H}_{2}$ gives rise to an opacity that is stronger in the infrared, its effects are dramatic in $V-K$.

The $J H K$ color-color diagram (Fig. 7) reveals the shape of the SED in the infrared and is a most useful diagnostic of atmospheric composition of cool white dwarfs $\left(T_{\text {eff }} \lesssim 4500\right.$ $\mathrm{K})$. In this diagram, both the pure $\mathrm{H}$ and pure $\mathrm{He}$ sequences are insensitive to gravity. This confines atmospheres with pure compositions to two well-separated curves. Pure $\mathrm{He}$ atmospheres with $T_{\text {eff }} \lesssim 4500 \mathrm{~K}$ are confined to the upper right region of the diagram where $H-K>0.15$ and $J-H>0.25$, and their colors become redder for lower $T_{\text {eff }}$. On the other hand, pure $\mathrm{H}$ atmospheres have bluer colors with $H-K<0.05$ and $-0.2<J-H<0.25$. The overlap of mixed $\mathrm{H} / \mathrm{He}$ atmospheres with the pure composition atmospheres is very limited in the $J H K$ diagram, and stars with $J-H<-0.25$ most certainly have mixed composition atmospheres.

Finally, we note that Bergeron et al. (1997) discuss the possibility of a continuum opacity source in pure $\mathrm{H}$ atmospheres that would affect the $U$ and $B$ bands. This helps in reproducing the $B-V$ color of the most extreme stars in their sample (Fig. 6, three uppermost stars). We have not included this opacity source in our models, however. The spectral fits described below were performed both with and without the $U$ and $B$ photometry; nearly identical results were obtained.

It is not presently possible to derive accurate ages for very cool white dwarfs with pure $\mathrm{He}$ or He-rich atmospheres. However, as a point of reference we include in Table 2 ages of extremely cool, pure $\mathrm{H}$ white dwarfs. Correct treatments of helium pressure ionization, which determines the location of the photosphere of these objects and of $\mathrm{He}^{-}$absorption cross sections at high densities, are lacking and prevent the derivation of reliable cooling curves for mixed composition objects. The only certainty is that, because of their more transparent atmospheres, white dwarfs with He-rich atmospheres cool faster than their H-rich counterparts. For example, current models (Chabrier 1997) allow us to estimate that a pure He atmosphere, $0.6 M_{\odot}$ white dwarf will reach an effective temperature $T_{\text {eff }}=4000 \mathrm{~K}$ after only $\sim 6 \mathrm{Gyr}$, much faster than cool DA white dwarfs. One should further note that even a small admixture of hydrogen or metals, due to either accretion or internal mixing processes, will slow down the cooling of the object dramatically, bringing it closer to a DA cooling sequence.

\section{1. $W D 0346+246$}

Figure 5 reveals that WD $0346+246$ is intrinsically the faintest (in the $V$ band) white dwarf known, with the exception of the high-mass degenerate ESO 439-26 (Ruiz et al. 1995). While it is at the fork between the $\mathrm{H}$ and He cooling sequences, it does not obviously belong to either. Its $B V K$ colors (Fig. 6) reveal that it definitely does not have a pure $\mathrm{He}$ atmosphere. It does not sit on the pure $\mathrm{H}$ models either, except possibly with a very low gravity of $\log g \approx 6.5$ and $T_{\text {eff }} \approx 2700 \mathrm{~K}$. Indeed, a fit of pure hydrogen models to the broadband photometry obtained by minimizing the errorweighted $\chi^{2}$ gives $T_{\text {eff }}=2790 \mathrm{~K}$ for $\log g=6.5$ (Fig. 8, solid line). Since the distance of WD $0346+246$ is known, the fit directly gives the radius of the star, $R=0.0174 R_{\odot}$. While our fit is fairly good, it is problematic in several ways. First, the optimal $\chi^{2}$ and $T_{\text {eff }}$ decrease slowly for even lower gravities. Such low values of $T_{\text {eff }}$ and gravity would be rather extreme parameters for a white dwarf. Second, the slope of the fitted spectrum in the infrared is rather shallow, and it misses the $J$ and $K$ photometry by 3-4 $\sigma$. Indeed, the $J-H$ color of WD $0346+246$ (Fig. 7) is $\sim 0.5 \mathrm{mag}$ bluer than both the sequences of pure $\mathrm{H}$ and pure He models. Third, the model spectrum predicts a strong CIA depression near 1 $\mu \mathrm{m}$, which is not present in the observed spectrum (Fig. 1), indicating that pure hydrogen models are inappropriate.

We think that it is far more likely that the atmosphere of WD $0346+246$ is of mixed hydrogen and helium composition. The colors of WD $0346+246$ can be interpreted as those of a mixed $\mathrm{H} / \mathrm{He}$ atmosphere based on Figs. 5-7. This is particularly supported by its $J-H$ color (Fig. 7). Using a very limited grid of mixed composition atmospheres with $T_{\text {eff }}=3500$ and $3750 \mathrm{~K}, \log g=8$, and $0 \leq y \leq 10^{7}$, we have fitted the He abundance to the observed broadband fluxes of WD $0346+246$. The result is shown by the dotted line in Figure 8. The fit of the photometry is excellent, and the synthetic spectrum agrees remarkably well with our observed spectrum (Fig. 1) at all wavelengths. We obtain a

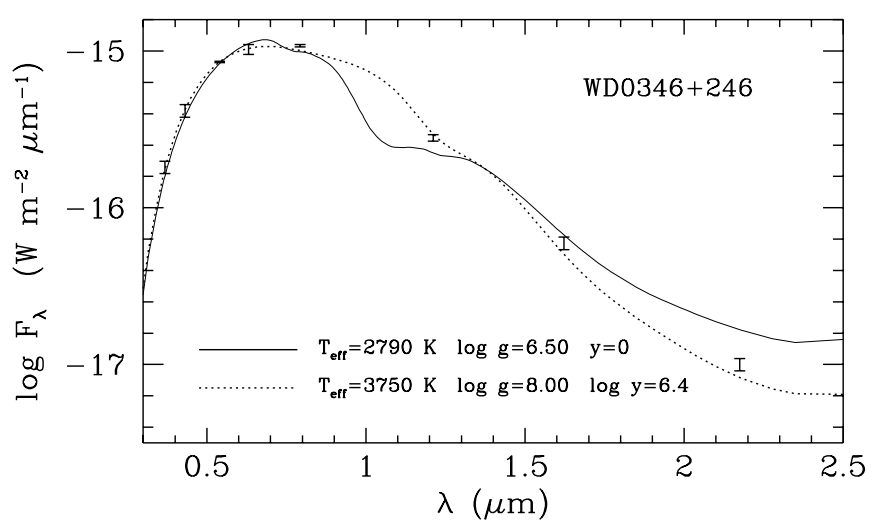

Fig. 8.-Fit of the $U$ through $K$ photometry of WD $0346+246$. The broadband photometric measurements (Table 1 ) are shown by $\pm 1 \sigma$ error bars. The solid curve indicates the best-fit pure hydrogen atmosphere model with a gravity of $\log g=6.5$. A slightly better fit can be obtained at even lower gravities. The dotted curve is the best-fit mixed $\mathrm{H} / \mathrm{He}$ atmosphere. 
helium abundance of $\log y \approx 6.4$. Because the models are rather crude, this determination is inaccurate, but it clearly indicates that the atmosphere contains only traces of hydrogen. The fitted radius is $R=0.010 \pm 0.002 R_{\odot}$. Finally, our choices of $T_{\text {eff }}$ and $\log g$ for the mixed composition sequence appear to be reasonable estimates for WD $0346+246$. From our analysis based on model atmospheres and spectra, we conclude that WD $0346+246$ is a very cool white dwarf with $T_{\text {eff }} \approx 3700 \mathrm{~K}$, a typical surface gravity of $\log g \approx 8$, and a helium-dominated atmosphere $(5 \lesssim$ $\log y \lesssim 8)$.

\subsection{LHS 3250}

LHS 3250, on the other hand, stands apart from all other known white dwarfs in Figures 5-7. We can say with certainty that it does not have a pure $\mathrm{He}$ atmosphere. Its loci in each of these figures lead to contradictory interpretations. Figure 5 suggests that it is a very $\operatorname{cool}\left(T_{\text {eff }} \approx 2400 \mathrm{~K}\right)$, pure $\mathrm{H}$ or mixed $\mathrm{H} / \mathrm{He}$ white dwarf. Its high $V$-band luminosity indicates that it has either a very low gravity and low $T_{\text {eff }}$ or a high gravity with very high $T_{\text {eff }}$, or it may be a double degenerate. We can immediately exclude the hightemperature possibility: its $B V R$ colors are identical to those of WD 1656-062, which has a pure $\mathrm{H}$ atmosphere, $T_{\text {eff }}=5520 \mathrm{~K}$, and $\log g=8$ (Bergeron et al. 1997). If it were this hot, however, it would show detectable $\mathrm{H} \alpha$ absorption, but it does not. Furthermore, its infrared colors would not be blue. Its $B V K$ colors (Fig. 6) can be understood if $T_{\text {eff }} \approx 4200 \mathrm{~K}$ with a mixed $\mathrm{H} / \mathrm{He}$ atmosphere dominated by $\mathrm{He}(\log y \gtrsim 1$; see also Fig. 15 of Bergeron et al. 1995a). Its infrared colors (Fig. 7) suggest that it has a mixed $\mathrm{H} / \mathrm{He}$ atmosphere dominated by He but with a very low temperature. Finally, our spectrum is featureless, and the absence of a steep drop in the flux near $1.0 \mu \mathrm{m}$ argues against significant $\mathrm{H}_{2}$ CIA but not against $\mathrm{He}-\mathrm{H}_{2}$ CIA. It seems that neither pure $\mathrm{H}$ nor pure $\mathrm{He}$ atmospheres are sufficient to understand the nature of LHS 3250. Furthermore, Harris et al. (1999) were not able to fit its $V$ through $K$ photometry with either pure $\mathrm{H}$ or mixed $\mathrm{H} / \mathrm{He}$ atmospheres. We have also failed with our new models, and its SED remains unexplained.

A feasible solution to this problem may be that LHS 3250 is an unequal mass binary, with one component similar to WD $0346+246$ and the other much cooler. This would have the effect of amplifying the optical flux, as compared to a single white dwarf, while contributing minimal amplification in the infrared. Until the full grid of mixed $\mathrm{H} / \mathrm{He}$ models has been calculated, a synthetic, composite spectrum of such a combination is not forthcoming. We can only conclude that $T_{\text {eff }}$ is below $4000 \mathrm{~K}$, and, based on the severity of the $0.6-2.5 \mu \mathrm{m}$ absorption, we suggest that it must be cooler than WD $0346+246$ with $T_{\text {eff }} \approx 3700 \mathrm{~K}$.

\section{3. $F 351-50$}

We have analyzed the $0.35-1.05 \mu \mathrm{m}$ spectrum of F351 - 50 obtained by Ibata et al. (2000). Unfortunately, this part of the spectrum is not very sensitive to differences in surface composition. However, the presence of CIA, and hence of a significant amount of $\mathrm{H}$, is suggested by the apparent change in the slope of the spectrum for $\lambda>0.95$ $\mu \mathrm{m}$. Although this is a very noisy part of the spectrum, we obtain an excellent fit with pure hydrogen models (Fig. 9). The fitted parameters are $T_{\text {eff }}=2844 \mathrm{~K}$ and $\log g=6.5$. These are extreme and unlikely parameters for a white

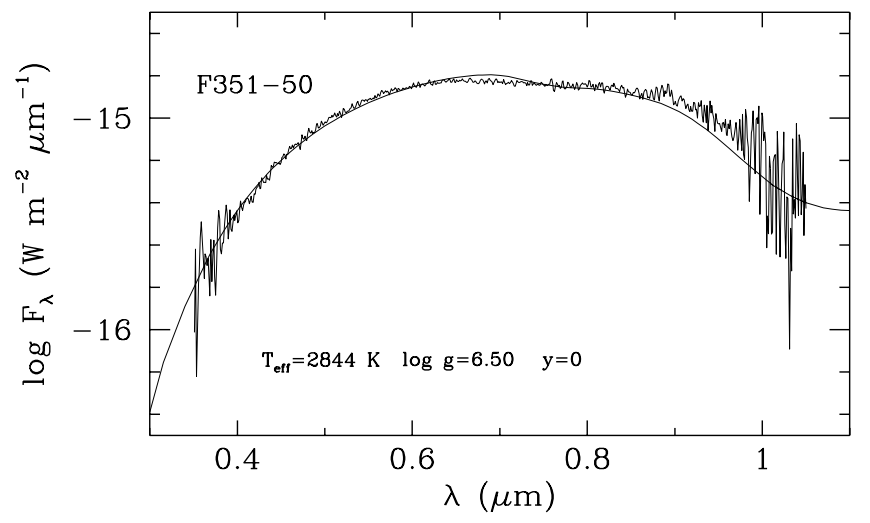

FIG. 9.-Fit of the spectrum of F351-50 by Ibata et al. (2000) with a pure hydrogen atmosphere

dwarf, and we attach only modest significance to the result, for the same reasons that a similar fit to WD $0346+246$ was not adequate.

The $B V R I$ colors of F351-50 are nearly identical to those of WD $0346+246$, suggesting that it may have a similar composition. If we ignore the part of the spectrum beyond $0.95 \mu \mathrm{m}$, where the $\mathrm{S} / \mathrm{N}$ declines dramatically, we obtain an equally good fit with our restricted grid of mixed $\mathrm{H} / \mathrm{He}$ composition atmospheres with $T_{\text {eff }}=3500 \mathrm{~K}$, $\log g=8$, and $\log y=5.85$. As in the case of WD $0346+246$, we find that mixed composition models lead to less extreme physical parameters. A more definitive analysis will require infrared photometry or the parallax of F351 - 50.

\section{CONCLUSION}

WD $0346+246$ appears to be the first very cool white dwarf with an atmosphere of mixed hydrogen and helium composition. It has an effective temperature below $4000 \mathrm{~K}$ and shows only traces of hydrogen in its atmosphere. Only three other cool white dwarfs are known to have significantly high $N(\mathrm{He}) / N(\mathrm{H})$ ratios. All three are in the $\mathrm{C}_{2} \mathrm{H}$ spectral class and have $T_{\text {eff }}>5300 \mathrm{~K}$ (Bergeron et al. 1997). Although the spectral coverage of the available data is more limited for F351-50, it appears to be very similar to WD $0346+246$. These two stars are the first examples of very cool white dwarfs with extremely high $\mathrm{He}$ to $\mathrm{H}$ mixing ratios and may provide important clues in understanding the spectral evolution of old white dwarfs.

The computation of an extensive and physically sound grid of mixed composition atmospheres for $T_{\text {eff }} \lesssim 5000 \mathrm{~K}$ is necessary to obtain reliable determinations of the surface parameters for WD $0346+246$ and F351-50. Such a grid of models may provide a better understanding of the intriguing white dwarf LHS 3250, which remains inscrutable.

We thank S. R. Kulkarni and J. S. Bloom for their support of this work, M. Irwin for providing us with his spectral data on F351-50, I. N. Reid for sharing the spectrum of LHS 3250, and J. Liebert for useful discussions and suggestions. We also graciously thank the two referees for a thorough job and for insisting that we check the technique in $\S 5$. Financial assistance for this work was provided by NASA through Hubble Fellowship grant HF-01122.01-99A from the Space Telescope Science Institute, which is oper- 
ated by the Association of Universities for Research in Astronomy, Inc., under NASA contract NAS5-26555. B. R. O. acknowledges FUTDI. D. S. and A. V. F. are supported by NSF grants AST 97-31438 and AST 99-87438, respectively. S. T. H. acknowledges the support of the Particle Physics and Astronomy Research Council. A. L. C. is grateful for a National Science Foundation Graduate Research Fellowship. Part of this work was done under the auspices of ALLIANCE project 00193 RL between the United
Kingdom and France. The William Herschel Telescope is operated on the island of La Palma by the Isaac Newton Group in the Spanish Observatorio del Roque de los Muchachos of the Instituto de Astrofisica de Canarias. The W. M. Keck Observatory is operated as a scientific partnership among the California Institute of Technology, the University of California, and NASA; it was made possible by the generous financial support of the W. M. Keck Foundation.
Bergeron, P., Ruiz, M.-T., \& Leggett, S. K. 1997, ApJS, 108, 339

Bergeron, P., Ruiz, M.-T., Leggett, S. K., Saumon, D., \& Wesemael, F. 1994, ApJ, 423, 456

Bergeron, P., Saumon, D., \& Wesemael, F. 1995a, ApJ, 443, 764

Bergeron, P., Wesemael, F., \& Beauchamp, A. 1995b, PASP, 107, 1047

Borysow, A., \& Frommhold, L. 1990, ApJ, 348, L41

Chabrier, G. 1997, in IAU Symp. 189, Fundamental Stellar Properties, ed. T. R. Bedding, A. J. Booth, \& J. Davis (New York: Kluwer), 381

Chabrier, G., Brassard, P., Fontaine, G., \& Saumon, D. 2000, ApJ, 543, 216

Filippenko, A. V. 1982, PASP, 94, 715

Hambly, N. C., Smartt, S. J., \& Hodgkin, S. T. 1997, ApJ, 489, L157

Hambly, N. C., Smartt, S. J., Hodgkin, S. T., Jameson, R. F., Kemp, S. N., Rolleston, W. R. J., \& Steele, I. A. 1999, MNRAS, 309, L33

Hansen, B. 1999, Nature, 394, 860

Harris, H. C., Dahn, C. C., Vrba, F. J., Henden, A. A., Liebert, J., Schmidt, G. D., \& Reid, I. N. 1999, ApJ, 524, 1000

\section{REFERENCES}

Hodgkin, S. T., Oppenheimer, B. R., Hambly, N. C., Jameson, R. F., Smartt, S. J., \& Steele, I. A. 2000, Nature, 403, 57

Ibata, R., Irwin, I., Bienaymé, O., Scholz, R., \& Guibert, J. 2000, ApJ, 532, L41

Landolt, A. U. 1992, AJ, 104, 340

Leggett, S. K., Ruiz, M.-T., \& Bergeron, P. 1998, ApJ, 497, 294

Matthews, K., \& Soifer, B. T. 1994, in Infrared Astronomy with Arrays: The Next Generation, ed. I. S. McLean (New York: Kluwer), 239

Oke, J. B., et al. 1995, PASP, 107, 375

Oke, J. B., \& Gunn, J. E. 1983, ApJ, 266, 713

Ruiz, M. T., Bergeron, P., Leggett, S. K., \& Anguita, C. 1995, ApJ, 455, L159

Saumon, D., \& Jacobson, S. 1999, ApJ, 511, L107 